\title{
Consórcios públicos de saúde: uma revisão da literatura
}

\author{
Public Health consortia: a literature review
}

Raquel Gonçalves Coimbra Flexa (https://orcid.org/0000-0001-9422-7895) ${ }^{1}$

Rafael Garcia Barbastefano (https://orcid.org/0000-0001-8253-6308) ${ }^{1}$

${ }^{1}$ Departamento de Engenharia de Produção, Centro Federal de Educação Tecnológica Celso Suckow da Fonseca. Av Maracanã 229, Maracanã. 20271-110 Rio de Janeiro RJ Brasil. raquelflexa@yahoo.com

\begin{abstract}
Regional Health Planning is a health services' hierarchization strategy that allows actions and services decentralization. Nonetheless, some challenges to implementing regional health planning are identified, such as difficulties in coordinating actions and services in different geographic locations, with different managements to meet population health needs with adequate scale, quality, and cost. In this context, intercity health consortia emerge as an organizational solution that allows better coordination and integration between federative entities, and their main benefits are scale gain in public services delivery; rationalization of processes and expenses; and realization of joint projects that would be impossible to be implemented if managed and funded in isolation. This paper aims to understand how health consortia reach better performance in the procurement and hiring of services by cooperative action through a narrative literature review. The results are organized into three parts: i) definition and concepts of public consortium; ii) definition and concepts of intercity health consortium (CIS); iii) health consortium case studies in Brazil.
\end{abstract}

Key words Health consortia, Regional health planning, Decentralization, Local health systems
Resumo A regionalização é uma estratégia de hierarquização dos serviços de saúde que orienta a descentralização das ações e serviços. Existem, entretanto, desafios para efetivar a regionalização, tais como as dificuldades para integrar e coordenar as ações e serviços, em diferentes espaços geográficos, com distintas gestões para atender as necessidades de saúde na escala, qualidade e custos adequados. Nesse contexto, surgem os consórcios públicos enquanto solução organizacional para suprir as necessidades de coordenação e integração entre os entes federativos. Os benefícios dos consórcios são o ganho de escala na prestação de serviços, a racionalidade de processos e de despesas, a realização de projetos conjuntos que seriam inviáveis de forma isolada. O objeto deste artigo é realizar uma investigação sobre como os consórcios intermunicipais de saúde conseguem obter melhor desempenho no que tange aos processos de aquisição e contratação de serviços de forma cooperativa, através de uma revisão bibliográfca narrativa. Os resultados da revisão foram divididos em 3 blocos: i) definições e conceitos dos consórcios públicos, ii) definições e conceitos sobre consórcios intermunicipais de saúde (CIS), iii) estudos de caso de CIS no Brasil.

Palavras-chaves Consórcios de saúde, Regionalização, Descentralização, Sistemas locais de saúde 


\section{Introdução}

Sistemas de Saúde podem ser definidos a partir de suas leis e regulações, forma de financiamento, lógicas de administração e o escopo dos serviços para o atendimento a determinada população ${ }^{1}$. Biassoto Júnior et al. ${ }^{2}$ afirmam que são amplas as discussões sobre a construção de sistemas de saúde universais, equânimes e integrais e que garantam o acesso da população a serviços resolutivos e de qualidade. No Brasil, saúde tem se mantido nos últimos anos como primeiro problema mais importante enfrentado no cotidiano pela população, sendo o subfinanciamento público do setor de saúde um dos fatores impeditivos para o cumprimento dos preceitos constitucionais de acesso universal e atendimento integral ${ }^{3}$.

Na IX Conferência Nacional de Saúde, discutiu-se a descentralização e a municipalização da saúde no Brasil. Para Buss ${ }^{4}$, o município, como célula mínima de estrutura político-administrativa brasileira, é teoricamente capaz de organizar um sistema de saúde mais adequado às necessidades de seus habitantes, por encontrar-se mais próximo do espaço concreto onde vivem as pessoas e mais sensível às pressões e reivindicações da população. O autor destaca, entretanto que há a necessidade do desenvolvimento institucional dos municípios, para que então seja possível darlhes competência para a formulação e execução dessas políticas sociais.

Para Souto Júnior ${ }^{5}$, a regionalização pressupõe uma forma de organização do SUS com base populacional e territorial, que busque uma distribuição de serviços e promova equidade do acesso, qualidade, otimização dos recursos e racionalidade dos gastos. Kehrig et al. ${ }^{6}$ reforçam que a constituição de redes de atenção regionalizada à saúde faz-se mediante pactuação entre os gestores envolvidos, para definição das responsabilidades compartilhadas ou não, através, por exemplo, das Comissões Intergestores Regionais (CIR).

Entretanto, apesar dos benefícios, existem ainda desafios para efetivar a regionalização, tais como as dificuldades para integrar e coordenar as ações e serviços, em diferentes espaços geográficos, com distintas gestões, para atender as necessidades de saúde e demandas da população na escala, qualidade e custos adequados ${ }^{7}$. Além disso, essa diretriz também implica na fragmentação na prestação de serviços e redução do poder de barganha nos processos de aquisição de materiais e serviços.

No contexto de restrições orçamentárias na gestão pública e necessidade de ampliação da efi- ciência do uso dos recursos públicos, associado à complexidade de gestão do SUS em um sistema federalista e com políticas explícitas de descentralização e regionalização, surgem os consórcios públicos enquanto solução organizacional para suprir as necessidades de coordenação e integração entre os entes federativos. Os benefícios dos consórcios são o ganho de escala na prestação de serviços públicos, a racionalidade de processos e de despesas, a realização de projetos conjuntos considerados inviáveis de forma isolada, além do fortalecimento político de regiões até então com pouca representatividade estadual ${ }^{8}$.

No que tange aos processos de aquisição de materiais e contratação de serviços, os consórcios têm mostrado melhor desempenho frente às compras individualizadas em cada um dos entes federativos. Amaral e Blatt ${ }^{9}$, ao estudarem o Consórcio Intermunicipal de Saúde do Vale do Itajaí (Santa Catarina), observaram uma redução aproximada de $48,0 \%$ no número de itens que apresentaram falta em pelo menos um dia, entre 2007 e 2009. Ferraes e Cordoni Júnior ${ }^{10}$ focaram sua análise no Consórcio Paraná Saúde e perceberam que, em 2000, a aquisição através desse ente apresentou um custo $29,7 \%$ inferior aos preços divulgados no Banco de Preços do Ministério da Saúde.

Dados os pontos expostos, o objetivo deste artigo é realizar uma investigação sobre como os consórcios intermunicipais de saúde conseguem obter melhor desempenho no que tange aos processos de aquisição e contratação de serviços de forma cooperativa, através de uma revisão bibliográfica narrativa.

Para isso, a presente publicação se apresenta em 3 partes: i) método utilizado para a busca bibliográfica; ii) exposição dos principais conteúdos extraídos através da leitura dos artigos, dissertações, teses e demais documentos levantados; iii) conclusões e comentários finais.

\section{Método}

Para Gil ${ }^{11}$, a pesquisa bibliográfica pode ser entendida como um processo que envolve as seguintes etapas: escolha do tema; levantamento bibliográfico preliminar; formulação do problema; elaboração do plano provisório de assunto; busca das fontes; leitura do material; fichamento; organização lógica do assunto; e redação do texto.

As revisões podem ser de três tipos: sistemáticas, narrativas e integrativas. Esse trabalho pode 
ser caracterizado como uma revisão narrativa. Para Rother ${ }^{12}$, os artigos de revisão narrativa são publicações amplas, apropriadas para descrever e discutir o desenvolvimento ou o estado da arte de um determinado assunto, sob ponto de vista teórico ou contextual. Segundo a autora, esse tipo de pesquisa é constituído pela análise da literatura publicada em livros, artigos de revista, e na interpretação e análise crítica pessoal do autor.

O método para a realização da revisão bibliográfica nessa pesquisa está sintetizado na Figura 1.

Nessa pesquisa buscou-se a utilização de bases especializadas em saúde, tanto no Brasil como com escopo internacional, além de bases de periódicos que reúnem diversos assuntos além da saúde pública.

A base especializada em saúde no Brasil escolhida foi a Biblioteca Virtual em Saúde (BVS) e a de escopo internacional foi a PubMed. Quanto às bases de dados não especializadas, optou-se por utilizar a ISI Web of Knowledge e Scopus, por conter os periódicos com maior impacto bibliométrico do mundo.

$\mathrm{Na}$ busca realizada na base BVS, o objetivo foi a busca de artigos, dissertações de mestrado, teses de doutorado e relatórios técnicos que não estivessem indexados pelas bases ISI, Scopus ou PubMed, e que tivessem um "olhar" específico para a realidade do Brasil. Nessa base, foram usadas as palavras-chave "consórcio" e "saúde" e o resultado obtido, sem restrição quanto ao ano de publicação, foi de 173 publicações. Dado que se trata de um tema de grande dinamicidade, optou-se por restringir a seleção para publicações posteriores ao ano 2000. Após esse novo filtro, restaram 113 trabalhos. Após a leitura dos resumos desses trabalhos, com o propósito de reconhecimento sobre o objeto detalhado daquelas publicações e verificação de adequação ao objetivo específico dessa pesquisa, relacionado com o desempenho na aquisição de materiais e contratação de serviços pelos consórcios, verificou-se que apenas 20 publicações se mostravam aderentes a essa pesquisa.

$\mathrm{Na}$ base Scopus, a primeira tentativa foi realizada com as palavras-chave "health" e "consortium”, sem restrição quanto à data de publicação. Retornaram 7.005 trabalhos que tratavam sobre o tema e, ao realizar um novo filtro para pesquisas posteriores ao ano 2000, esse resultado caiu para 5.918 publicações. Com um número ainda elevado, optou-se por adicionar mais uma palavra-chave: "purchasing". Essa escolha se deu pelo fato de que o foco dessa pesquisa é a compreensão sobre como os consórcios podem ser um instrumento para a obtenção de melhor desempenho em processos de aquisição. Após esse novo filtro, chegou-se, então, a 74 artigos. Após a leitura dos resumos, verificou-se que apenas 10 trabalhos tinham relação direta com o objeto dessa pesquisa.

O mesmo mecanismo de busca foi aplicado na base ISI - Web of Knowledge. Para as palavras-chave "health" e "consortium", sem restrição quanto ao período de publicação, retornaram 3.688 artigos. Restringindo para o período entre 2000 e 2017, chegou-se a 3.292 trabalhos. Ao acrescentar a palavra-chave "purchasing", o número caiu para 19. Ao realizar a leitura dos resumos, verificou-se a existência de 4 publicações aderentes a essa pesquisa, mas 3 delas eram coincidentes com publicações encontradas na busca na base Scopus.

Ao submeter as mesmas palavras-chave na base PubMed, observou-se que os periódicos contidos nessa base se encontravam indexados por alguma das três bases anteriormente consultadas, retornando então publicações que já haviam sido analisadas nas buscas anteriores.

\section{Resultados}

Os resultados da revisão bibliográfica foram divididos em três blocos. No primeiro bloco são apresentadas definições, vantagens e desvantagens dos consórcios públicos no Brasil, sem o foco específico na área de saúde, mas para o entendimento de como esse instrumento apresenta vantagens para realização de projetos e processos de forma cooperativa entre os entes federativos. O segundo bloco se foca especificamente nos Consórcios Intermunicipais de Saúde (CIS), destacando suas definições, histórico de formação e principais desafios de gestão. O último bloco apresenta alguns casos de CIS no Brasil, com um olhar sobre seus principais resultados, em especial para aquisição de bens e para a contratação cooperativa de serviços, além das dificuldades para sua implementação, manutenção e gestão.

O Quadro 1 apresenta as publicações utilizadas nessa revisão narrativa, através da exposição de títulos, autores, ano de publicação, veículo de publicação e principais assuntos abordados. 


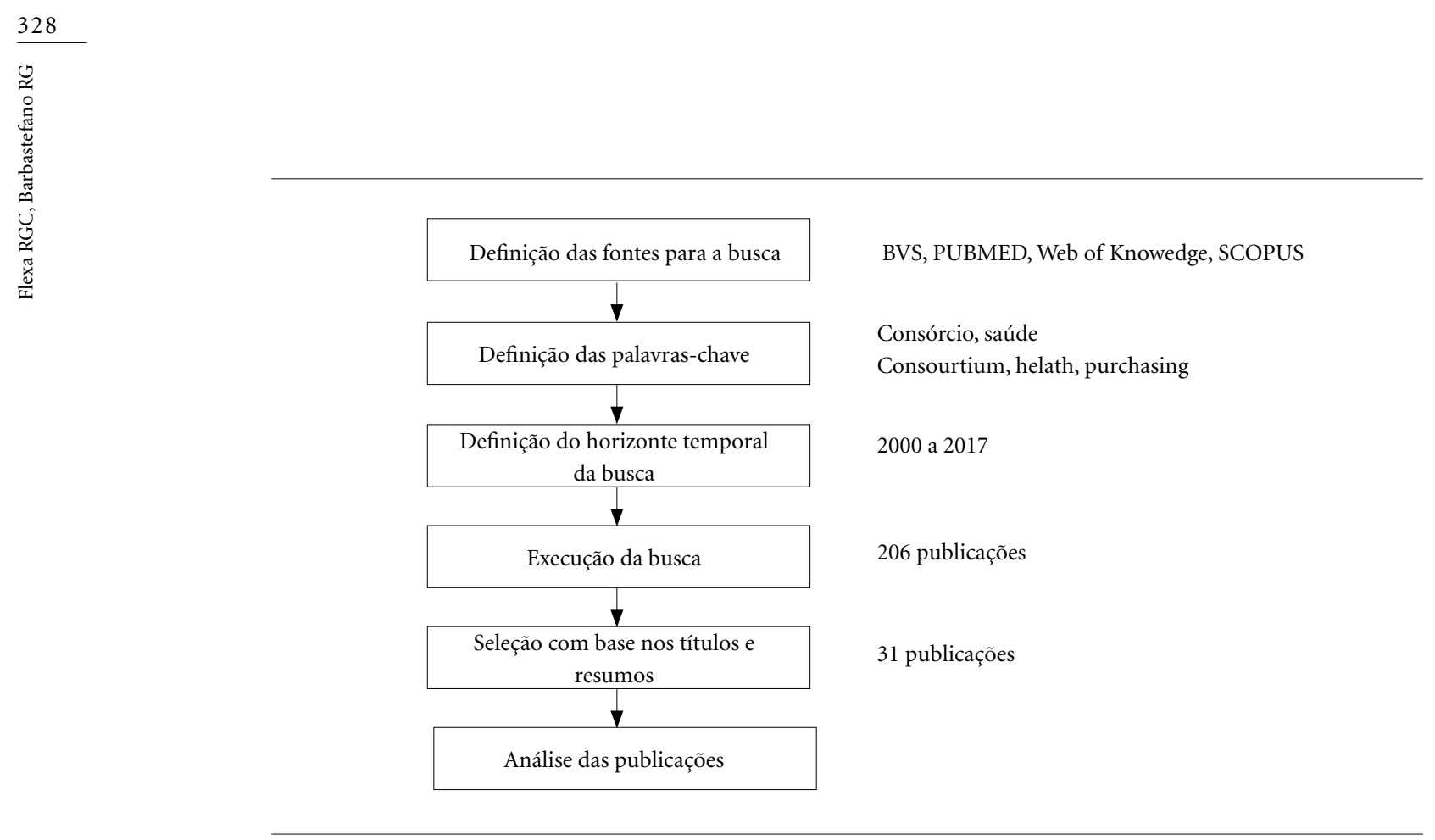

Figura 1. Método da revisão bibliográfica.

\section{Consórcios públicos: definições, vantagens e desvantagens}

O Observatório dos Consórcios Públicos e do Federalismo ${ }^{13}$ reconhece que a Constituição de 1988 consagrou o sistema federativo brasileiro baseado em um pacto que estabelece a autonomia das esferas territoriais de poder. Embora se verifiquem ganhos com o modelo federalista, a federação brasileira é caracterizada pela forte assimetria entre os governos, o que traz a necessidade de se planejar políticas capazes de corrigir ou minimizar as desigualdades entre estados e municípios $^{14}$.

Baldissera $^{8}$ afirma que o modelo de descentralização não foi acompanhado por instrumentos de planejamento e coordenação das ações governamentais e, nesse contexto, os consórcios intermunicipais surgiram como um mecanismo de coordenação que se localizam em esferas que extrapolam os limites territoriais dos municípios e, ao mesmo tempo, não alcançam os limites dos estados. Os consórcios públicos podem ser entendidos enquanto uma solução organizacional para suprir as necessidades de coordenação e integração entre os entes federativos, destinada a operar competências a ele delegadas, constituindo-se como expressão do exercício das autonomias dos entes consorciados ${ }^{15}$.

Trata-se de um instrumento de cooperação técnica e financeira entre municípios, estados e a
União, que pode servir para a articulação de ativos, viabilizar cooperação em projetos de abrangência regional, obras e outras ações destinadas a promover o desenvolvimento de determinada região ${ }^{16}$.

Embora a Lei $11.107 / 2005^{17}$, ou Lei dos Consórcios, tenha institucionalizado a figura dos consórcios públicos no Brasil, apenas em 2005 verifica-se a existência de mecanismos de consorciamento anteriores a essa data ${ }^{18}$. Nessas estruturas, os grupos de entes federados, majoritariamente municípios, associavam-se de forma horizontal e cooperada e decidiam agir de forma conjunta, sem fins lucrativos, para solucionar problemas comuns em determinada área, sem personalidade jurídica, possuindo apenas um protocolo de intenções.

Levando-se em conta o princípio de economicidade, mesmo com a capacidade de prestar o serviço de forma isolada, pode ser vantajoso buscar parceria com outros municípios, gastando-se menos recursos e obtendo-se os mesmos resultados. Pode-se também aumentar a qualidade dos serviços prestados mantendo-se os gastos que seriam incorridos de forma isolada ${ }^{8}$.

Linhares et al. ${ }^{19}$ observam que os consórcios se mostram instrumentos de grande valia na atuação em políticas que visam solucionar problemas que ocorrem em territorialidades multimunicipais, como saúde, meio ambiente e infraestrutura. 
Quadro 1. Quadro resumo dos artigos utilizados na revisão narrativa.

\begin{tabular}{|c|c|}
\hline Título & Principais assuntos abordados \\
\hline $\begin{array}{l}\text { Physician-Owned Surgical Hospitals } \\
\text { Outperform Other Hospitals in Medicare } \\
\text { Value-Based Purchasing Program }{ }^{20}\end{array}$ & $\begin{array}{l}\text { Medição do desempenho de aquisicão dos hospitais do Medicare que } \\
\text { aderiram ao "Hospital Value-Based Purchasing Program" }\end{array}$ \\
\hline $\begin{array}{l}\text { Medical technology procurement in } \\
\text { Europe: A cross-country comparison of } \\
\text { current practice and policy }\end{array}$ & $\begin{array}{l}\text { Estudo sobre a influência das políticas de compras para a difusão do uso } \\
\text { de produtos para a saúde em sistemas universais de saúde na Europa }\end{array}$ \\
\hline $\begin{array}{l}\text { Hospital purchasing alliances: Utilization, } \\
\text { services, and performance }{ }^{22}\end{array}$ & $\begin{array}{l}\text { Estudos sobre como consórcios de hospitais conseguiram reduzir os } \\
\text { custos de aquisição de produtos, bem como os custos de transação, em } \\
\text { particular no caso de medicamentos. }\end{array}$ \\
\hline $\begin{array}{l}\text { Case study of how successful coordination } \\
\text { was achieved between a mental health and } \\
\text { social care service in Sweden }{ }^{23}\end{array}$ & $\begin{array}{l}\text { Estudo sobre a formação e a estrutura de coordenação de um consórcio } \\
\text { para provimento de serviços de saúde mental na Suécia }\end{array}$ \\
\hline $\begin{array}{l}\text { Optimal administrative geographies: An } \\
\text { algorithmic approach }{ }^{24}\end{array}$ & $\begin{array}{l}\text { Proposição de elementos para auxiliar a decisão de definição dos } \\
\text { territórios administrativos de saúde no Reino Unido, tais como: } \\
\text { densidade demográfica, coexistência de autoridades locais no território, } \\
\text { tamanho e homogeneidade da população atendida }\end{array}$ \\
\hline $\begin{array}{l}\text { Impact of Supply Chain Collaboration on } \\
\text { Value Co-creation and Firm Performance: } \\
\text { A Healthcare Service Sector Perspective }{ }^{25}\end{array}$ & $\begin{array}{l}\text { Conceituação de colaboração na cadeia de suprimentos no setor de saúde, } \\
\text { com foco nas cadeias de suprimentos de hospitais }\end{array}$ \\
\hline New Trends in Healthcare Supply chain ${ }^{26}$ & $\begin{array}{l}\text { Artigo sobre novas tendências para redução de custos na cadeia } \\
\text { de suprimentos do setor de saúde, com uso de ferramentas como } \\
\text { centralização virtual das cadeias de suprimentos, práticas de gestão da } \\
\text { cadeia de suprimentos, tecnologia RFID, analise de dados, entre outros. }\end{array}$ \\
\hline $\begin{array}{l}\text { Building a Better Delivery System: A New } \\
\text { Engineering/Health Care Partnership }\end{array}$ & $\begin{array}{l}\text { Relatório com a identificação de aplicações de engenharia que podem } \\
\text { contribuir para o setor de saúde no curto, médio e longo prazo, além de } \\
\text { verificação de fatores que podem acelerar ou impedir a adoção dessas } \\
\text { tecnologias. }\end{array}$ \\
\hline $\begin{array}{l}\text { Learning for the NHS on procurement and } \\
\text { supplychain management: a rapid evidence } \\
\text { assessment }{ }^{28}\end{array}$ & $\begin{array}{l}\text { Coletânea sobre estudos no NHS sobre como custos do sistema } \\
\text { de saúde podem ser reduzidos através de melhoria da relação com } \\
\text { fornecedores, ampliação da competências e habilidades de compras, } \\
\text { uso de tecnologia para a gestão de materiais }\end{array}$ \\
\hline $\begin{array}{l}\text { Supply Chain for Regional Centralized } \\
\text { Purchasing: An Application } \text { Model }^{29}\end{array}$ & $\begin{array}{l}\text { Proposição de um modelo de compras centralizadas nos níveis } \\
\text { regionais e nacionais de sistemas de saúde europeus, com forma de } \\
\text { redução dos custos de aquisição }\end{array}$ \\
\hline $\begin{array}{l}\text { Conditional Cash Transfers for Improving } \\
\text { Uptake of Health Interventions in Low- } \\
\text { and Middle-Income Countries }{ }^{30}\end{array}$ & $\begin{array}{l}\text { Estudo sobre como o condicionamento de transferências de recursos } \\
\text { em função de desempenho pode ampliar o uso de serviços de saúde } \\
\text { preventivos e os indicadores finalísticos de saúde }\end{array}$ \\
\hline $\begin{array}{l}\text { Consórcios de saúde: estudo de caso } \\
\text { exitoso }^{31}\end{array}$ & $\begin{array}{l}\text { Estudo de caso sobre o CIS mais antigo do setor saúde (Consórcio } \\
\text { Intermunicipal de Saúde da Região de Penápolis - CISA) para elaborar } \\
\text { um conjunto de recomendações, com base na experiência analisada, } \\
\text { para adaptações a outras experiências de CIS. }\end{array}$ \\
\hline $\begin{array}{l}\text { Institucionalidade e governança da } \\
\text { regionalização da saúde: o caso da região } \\
\text { Sul Mato-Grossense à luz das atas do } \\
\text { colegiado de gestão }{ }^{6}\end{array}$ & $\begin{array}{l}\text { Discussão sobre a regionalização da saúde, objetivando analisar as } \\
\text { dimensões da institucionalidade e governança na sua gestão, entendida } \\
\text { através instâncias consolidadas CIR; central de regulação; consórcio } \\
\text { intermunicipal; e hospital regional. }\end{array}$ \\
\hline $\begin{array}{l}\text { Percepção dos gestores municipais de } \\
\text { saúde relacionada à saúde ambiental: } \\
\text { consórcio intermunicipal de saúde } \\
\text { Cerrado Tocantins Araguaia }{ }^{32}\end{array}$ & $\begin{array}{l}\text { O estudo prospectivo teve como objetivo analisar a percepção } \\
\text { e as ações dos gestores municipais que compõem o consórcio } \\
\text { intermunicipal Cerrado Araguaia Tocantins quanto aos problemas de } \\
\text { saúde ambiental da região Norte do estado }\end{array}$ \\
\hline
\end{tabular}


Quadro 1. Quadro resumo dos artigos utilizados na revisão narrativa.

\begin{tabular}{|c|c|}
\hline $\begin{array}{l}\text { Constituição de Consórcios } \\
\text { Intermunicipais de Saúde: Uma Aplicação } \\
\text { da Técnica de Agrupamento por Clusters } \\
33\end{array}$ & $\begin{array}{l}\text { O objetivo deste artigo é propor a utilização da técnica de } \\
\text { agrupamentos por clusters, aplicada aos dados de internação dos } \\
\text { municípios do Estado de São Paulo como uma tentativa de diminuir a } \\
\text { arbitrariedade na constituição de consórcios intermunicipais }\end{array}$ \\
\hline $\begin{array}{l}\text { Consórcio intermunicipal para a } \\
\text { aquisição de medicamentos: impacto no } \\
\text { desabastecimento e no custo }{ }^{9}\end{array}$ & $\begin{array}{l}\text { O estudo analisa o impacto de consórcio para a aquisição de } \\
\text { medicamentos no desabastecimento e no custo dos medicamentos } \\
\text { do Componente da Assistência Farmacêutica Básica do município de } \\
\text { Indaial, SC. }\end{array}$ \\
\hline $\begin{array}{l}\text { Cooperação intermunicipal, } \\
\text { reterritorialização da gestão pública e } \\
\text { provisão de bens e serviços sociais no } \\
\text { Brasil contemporâneo: a experiência dos } \\
\text { Consórcios de Saúde de Minas Gerais }{ }^{34}\end{array}$ & $\begin{array}{l}\text { Estudo para a verificação do impacto do consorciamento na ampliação } \\
\text { e melhoria da qualidade dos serviços de saúde, além dos seus } \\
\text { condicionantes de natureza política e administrativa nos consórcios do } \\
\text { Estado de Minas Gerais }\end{array}$ \\
\hline $\begin{array}{l}\text { Consórcio de medicamentos no Paraná: } \\
\text { análise de cobertura e custos }{ }^{10}\end{array}$ & $\begin{array}{l}\text { O artigo analisa a cobertura e custos do Consórcio Paraná Saúde } \\
\text { (CPS), constituído para aquisição de medicamentos para prefeituras do } \\
\text { Paraná. }\end{array}$ \\
\hline $\begin{array}{l}\text { Consórcios Intermunicipais de Saúde: } \\
\text { Uma Análise à Luz da Teoria dos } \operatorname{Jogos}^{35}\end{array}$ & $\begin{array}{l}\text { O estudo analisa a formação e a sustentabilidade político-financeira dos } \\
\text { Consórcios Intermunicipais de Saúde por meio de um jogo dinâmico } \\
\text { de dois períodos com informação imperfeita, no qual dois prefeitos de } \\
\text { municípios decidem quanto à adesão e à permanência no consórcio }\end{array}$ \\
\hline $\begin{array}{l}\text { Avaliação do processo de implementação } \\
\text { do Consórcio Intermunicipal de Saúde da } \\
\text { região do Teles Pires }\end{array}$ & $\begin{array}{l}\text { A dissertação avaliou o processo de implementação do Consórcio } \\
\text { Intermunicipal de Saúde da microrregião do Teles Pires, no contexto da } \\
\text { regionalização de atenção à saúde. }\end{array}$ \\
\hline $\begin{array}{l}\text { Cooperação intermunicipal no âmbito do } \\
\text { SUS }\end{array}$ & $\begin{array}{l}\text { A dissertação trata sobre as interações entre vários agentes que podem } \\
\text { estar envolvidos com a estruturação de uma cooperação intermunicipal } \\
\text { voltada à política pública de saúde. }\end{array}$ \\
\hline $\begin{array}{l}\text { Ensaios sobre consórcios intermunicipais } \\
\text { de saúde: Financiamento, Comportamento } \\
\text { Estratégico, Incentivos e Economia } \\
\text { Política }^{38}\end{array}$ & $\begin{array}{l}\text { Discussão sobre os Consórcios Intermunicipais de Saúde com base } \\
\text { em fundamentos teóricos propiciados pelas teorias das parcerias e da } \\
\text { competição eleitoral, aliados a modelos formais de teoria dos jogos e } \\
\text { teoria de contratos }\end{array}$ \\
\hline $\begin{array}{l}\text { A regionalização da saúde no Estado do } \\
\text { Mato Grosso: o processo de implementação } \\
\text { e a relação público-privada na região de } \\
\text { saúde do Médio Norte mato-grossense }{ }^{39} \\
\end{array}$ & $\begin{array}{l}\text { Caracterização dos instrumentos e mecanismos adotados na } \\
\text { regionalização no Médio Norte mato-grossense, com destaque para as } \\
\text { relações público-privadas no sistema público de saúde, além da discussão } \\
\text { sobre o processo decisório e interação entre os diversos atores }\end{array}$ \\
\hline $\begin{array}{l}\text { Cooperação Intergovernamental em busca } \\
\text { do Desenvolvimento Regional }{ }^{14}\end{array}$ & $\begin{array}{l}\text { O objetivo do trabalho é identificar de que forma a cooperação } \\
\text { intergovernamental pode colaborar para a elaboração de políticas } \\
\text { públicas integradas, através da investigação das contribuições das ações } \\
\text { transversais para o fortalecimento de municípios e macrorregiões }\end{array}$ \\
\hline $\begin{array}{l}\text { Desafios da adaptação à lei de consórcios } \\
\text { públicos: experiências concretas no Estado } \\
\text { de São Paulo }{ }^{40}\end{array}$ & $\begin{array}{l}\text { O artigo tem como objetivo apresentar um estudo a respeito do } \\
\text { processo de adaptação dos consórcios à Lei no } 11.107 / 2005 \text {, com ênfase } \\
\text { na investigação de consórcios no estado de São Paulo, que não se } \\
\text { converteram para consórcios públicos nos moldes da lei. }\end{array}$ \\
\hline $\begin{array}{l}\text { Consórcios intermunicipais paulistas rumo } \\
\text { aos consórcios públicos - reflexões }{ }^{41}\end{array}$ & $\begin{array}{l}\text { O objetivo do artigo foi identificar os consórcios existentes no Estado } \\
\text { de São Paulo e sua adequação à Lei Federal 11.107/2005, através do } \\
\text { levantamento nos } 645 \text { municípios paulistas, entre junho e setembro de } \\
2010 .\end{array}$ \\
\hline $\begin{array}{l}\text { O papel da CIB/MG no processo de } \\
\text { regionalização do SUS em Minas Gerais }\end{array}$ & $\begin{array}{l}\text { A dissertação descreve como a CIB/MG contribuiu para o processo de } \\
\text { regionalização em Minas Gerais, no período de } 2004 \text { a } 2007 .\end{array}$ \\
\hline $\begin{array}{l}\text { Avaliação da satisfação dos usuários com } \\
\text { os serviços do consórcio intermunicipal de } \\
\text { saúde do noroeste do Paraná }{ }^{42}\end{array}$ & $\begin{array}{l}\text { A fim de avaliar a satisfação dos usuários com os serviços prestados pelo } \\
\text { CISA do noroeste do Paraná, foi realizada entrevista com os usuários } \\
\text { do serviço Saúde. Concluiu-se que o CISA é uma referência para os } \\
\text { pequenos municípios e que os usuários estão satisfeitos com os serviços } \\
\text { prestados pelo mesmo. }\end{array}$ \\
\hline
\end{tabular}


Quadro 1. Quadro resumo dos artigos utilizados na revisão narrativa.

\begin{tabular}{|l|l|}
\hline $\begin{array}{l}\text { Municípios cooperando com municípios: } \\
\text { relações federativas e consórcios } \\
\text { intermunicipais de saúde no Estado de São } \\
\text { Paulo }^{43}\end{array}$ & $\begin{array}{l}\text { O trabalho analisa o Consórcio de Saúde do Alto Vale do Ribeira, } \\
\text { descrevendo seu desenho institucional e analisando os resultados e as } \\
\text { principais transformações ocorridas na rede de serviços dos municípios } \\
\text { consorciados, após sua implementação }\end{array}$ \\
\hline $\begin{array}{l}\text { Regionalização dos Serviços de Saúde } \\
\text { em Mato Grosso: um estudo de caso da } \\
\text { implantação do Consórcio Intermunicipal } \\
\text { de Saúde da Região do Teles Pires, no } \\
\text { período de } 2000 \text { a } 2008^{44}\end{array}$ & $\begin{array}{l}\text { O objetivo do artigo é avaliar a implantação do Consórcio Intermunicipal } \\
\text { de Saúde da Região do Teles Pires-MT, através da análise dos contextos } \\
\text { externos da região (sociodemográfico e epidemiológico) e assistencial do } \\
\text { consórcio (organização, financiamento e produção de serviços), com base } \\
\text { em dados secundários, no período 2000-2008. }\end{array}$ \\
\hline $\begin{array}{l}\text { Gestão interfederativa do SUS: a } \\
\text { experiência gerencial do Consórcio } \\
\text { Intermunicipal do Sertão do Araripe de } \\
\text { Pernambuco }{ }^{18}\end{array}$ & $\begin{array}{l}\text { O objetivo do artigo é analisar a experiência gerencial do Consórcio } \\
\text { Intermunicipal de Saúde do Sertão do Araripe de Pernambuco (Cisape), } \\
\text { através de um modelo de gestão gerencialista, ou seja, orientado pela } \\
\text { gestão pública pós-burocrática }\end{array}$ \\
\hline
\end{tabular}

A sustentabilidade dos consórcios exige um pacto político consistente entre os atores federativos, visando objetivos de longo prazo em detrimento do imediatismo, e que seja capaz de tratar com a diversidade em detrimento da decisão unilateral ${ }^{15}$

Ademais, percebe-se que as dificuldades encontradas pelos entes federativos são transportadas para os consórcios, como a baixa qualificação dos servidores públicos, conflitos de agenda entre as áreas, lacunas de planejamento, entraves burocráticos e recursos finitos e mal aplicados.

Apesar da flexibilidade gerada pelos consórcios, muitos enfrentam obstáculos para sua operação por causa da fragilidade jurídica; dificuldade na relação com outros entes federados; falta de compromisso e responsabilidade de pagamento das contribuições municipais; uso político do consórcio e irresponsabilidade fiscal ${ }^{41}$.

\section{Os Consórcios Intermunicipais de Saúde (CIS)}

A descentralização da política de saúde, prevista na Constituição de $1988^{45}$, gerou um aumento significativo na participação dos municípios na provisão dos serviços de saúde ${ }^{31}$. No entanto, percebe-se que a transferência de responsabilidades da União para os estados e municípios tem se deparado com dificuldades relacionadas com deficiências organizacionais e financeiras, bem como ineficiências por problemas de escala e escopo, causada pela fragmentação dos serviços ${ }^{38}$. A autora afirma que houve perda de escala produtiva, resultante do incremento no número de estabelecimentos e de equipamentos, o que gerou sistemas de saúde ineficientes e a redução da qualidade dos serviços prestados à população.

O grande número de pequenos municípios, com pouca ou nenhuma disponibilidade de serviços de média e alta complexidade, mostrou que a descentralização precisa ser acompanhada por estratégias de coordenação e cooperação intergovernamental ${ }^{6}$.

Para implementar a política de regionalização da saúde, cabe aos municípios incorporar formas inovadoras de gestão e organização de redes de serviços mediante construção coletiva institucional $^{6}$. Nesse sentido, Neves e Ribeiro ${ }^{31}$ afirmam que os consórcios intermunicipais de saúde (CIS) são arranjos organizacionais que têm sido objeto frequente de avaliação de resultados, impactos e mecanismos de implementação na comunidade acadêmica, dado que favorecem a construção de sistemas regionais pactuados e, por isso, facilitam a implementação das estratégias de descentralização para a regionalização e hierarquização do SUS na oferta de serviços.

Os CIS se apresentam como inovações do setor público para uma melhor gestão, de modo a prover serviços especializados e de maior densidade tecnológica à população, além de gerar ganhos ambientais e econômicos na provisão desses serviços públicos ${ }^{32}$. Consórcios de saúde visam à resolução de problemas de gestão e prestação de serviços em uma região específica, mediante a soma de esforços e a articulação de recursos físicos, financeiros e tecnológicos, na busca de soluções dos problemas compartilha$\operatorname{dos}^{34,36}$. Isso permite a viabilização financeira de investimentos e contribui para a otimização da rede de assistência. 
Teixeira et al. ${ }^{35}$ definem os consórcios intermunicipais de saúde como uma iniciativa autônoma de municípios localizados em áreas geográficas contíguas, que se associam para gerir e prover conjuntamente serviços especializados e de apoio diagnóstico de maior densidade tecnológica à população das municipalidades participantes. Muller e Greco ${ }^{42}$ destacam os consórcios como um instrumento para solucionar desafios locais, como a ampliação e o ordenamento da oferta de serviços, a contratação de serviços, a flexibilização da remuneração de profissionais com pagamento de incentivos, a otimização dos recursos regionais disponíveis, com o intuito de melhorar o acesso e a qualidade dos serviços. Os consórcios pactuam regras de financiamento de serviços e de acesso de clientelas com base em recursos dos municípios associados ${ }^{5}$.

Morais e Chaves $^{32}$ percebem que a maior parte dos municípios brasileiros, principalmente aqueles afastados dos grandes centros ou com baixos índices populacionais, apresentam dificuldade para a implementação do SUS em função da dificuldade de alocação de recursos humanos qualificados, acesso ao uso de tecnologias e inexistência de estruturas físicas adequadas. Derivado disso, Rocha e Faria ${ }^{34}$, ao analisarem os consórcios do Estado de Minas Gerais, verificaram que os municípios, atuando de forma conjunta, podem alcançar resultados positivos como o aumento da capacidade de realização, ampliação do atendimento ao cidadão, maior eficiência no uso dos recursos públicos e a realização de ações que seriam inacessíveis a uma única prefeitura.

Dessa forma, eles conseguem suprir as carências individuais como a escassez de recursos financeiros, tecnológicos e humanos necessários à implementação das políticas públicas. Nesse sentido, Neves e Ribeiro ${ }^{31}$ afirmam que os CIS se mostram como uma alternativa de organização capaz de superar as deficiências individuais de cada município na assistência à saúde, principalmente em consultas médicas de especialidade e exames de média e alta complexidade, bem como reduzir os aspectos competitivos do federalismo brasileiro.

Morais e Chaves ${ }^{32}$, ao estudarem o Consórcio Intermunicipal de Saúde do Cerrado Araguaia Tocantins, percebem que os consórcios intermunicipais ocupam uma posição estratégica na estrutura hierárquica organizacional dos municípios, dado que permitem o desenvolvimento de estratégias e práticas intersetoriais, além de terem a autonomia necessária para a construção de parcerias e realização de planejamento para além do limite político e administrativo dos municípios.
Os consórcios de saúde possibilitam a maior integração de redes regionais de assistência à saúde, ampliando a oferta de leitos e o acesso a redes de média e alta complexidade, além de fortalecer o sistema de regulação regional ${ }^{46}$. $\mathrm{O}$ autor ainda destaca que esse tipo de modelo possibilita o planejamento regional estratégico, através do mapeamento conjunto das demandas, estabelecimento de prioridades e oferta de assistência técnica visando à elaboração de projetos integrados. Permite ainda o encaminhamento de pleitos conjuntos de solicitação de recursos, além da gestão de contratos e convênios compartilhada. Rocha e Faria ${ }^{34}$ ressaltam que, no caso dos consórcios do Estado de Minas Gerais, a prestação de serviços de forma regionalizada evita a sobrecarga do município na construção de novas unidades de oferta de serviços ambulatoriais e hospitalares, na contratação de recursos humanos especializados e na aquisição de equipamentos de custos elevados, possibilitando uma melhor utilização da rede disponível.

Outra vantagem de adesão dos municípios a um CIS é a estruturação de uma rede de acordo com a capacidade instalada dos diversos atores, reduzindo assim a capacidade ociosa e otimizando o serviço e o referenciamento dos pacientes entre municípios ${ }^{31}$. Além disso, por serem pessoas jurídicas de direito privado, conseguem ter maior flexibilidade administrativa, permitindo assim a contratação de pessoal segundo a lógica de mercado, com pagamento de salários competitivos, inclusive oferecendo bônus por produtividade, o que tenderia a melhorar o desempenho dos profissionais e elevar a qualidade dos serviços oferecidos $^{32}$.

Os consórcios residem em uma lógica de federalismo cooperativo, no qual é possível otimizar e integrar as ações públicas e propiciar a adoção de políticas redistributivas ${ }^{34}$. Os autores ressaltam, no entanto, que há a possibilidade de resultados negativos, como a subordinação dos entes mais fracos aos mais fortes, a falta de definição clara da distribuição das responsabilidades e consequente falta de accountability do sistema.

Quanto ao surgimento e formação dos consórcios, Neves e Ribeiro ${ }^{31}$, observaram duas principais tendências. Na primeira delas, ocorre a livre associação entre municípios segundo suas características políticas e uma regionalização que obedece a essas características. No segundo caso, há a indução governamental pelos governos estaduais, através da criação de mecanismos e incentivos para que haja essa associação conforme suas preferências. Os autores percebem que os 
consórcios se formam prioritariamente em torno de municípios pequenos ou médios, para o provimento de assistência especializada e verificaram que, dos municípios que fazem parte de consórcios, $95 \%$ tem menos de cinquenta mil habitantes e $60 \%$ tem menos de dez mil habitantes. Adicionalmente, Teixeira ${ }^{38}$ percebe que a maior parte dos municípios consorciados (52\%) tem renda média entre o quinto e oitavo decil e isso demonstra que os municípios que se consorciam são, em geral, municípios com renda média a alta. No entanto, Oliveira ${ }^{43}$ afirma que o mecanismo de consorciamento não se difundiu igualmente entre os estados e municípios do Brasil. A Tabela 1 mostra o número de consórcios em cada um dos estados brasileiros.

Souto Júnior ${ }^{5}$, no caso dos consórcios do Estado de Minas Gerais, percebeu que a autonomia transferida aos pequenos municípios, com menos de 30.000 habitantes, gerou déficit na oferta dos serviços de saúde prestados à população em função da falta de estruturas administrativas suficientes e eficientes, ausência de técnicos e recursos humanos em geral, precariedade de investimentos. $\mathrm{O}$ autor destaca que a autonomia municipal começou a ser mais factível quando os municípios, por meio de acordos de cooperação, resolveram se consorciar para comprar serviços com maior escala e com escopo definido com base nas suas necessidades, garantindo maior economicidade.

Neves e Ribeiro ${ }^{31}$, no caso do Consórcio Intermunicipal de Saúde da Região de Penápolis (CISA), afirmam que a estrutura decisória dos consórcios está centrada em um Conselho de Prefeitos e o gerenciamento do consórcio é feito por um órgão executivo. Para seu financiamento, os autores destacam a cotização dos municípios para a manutenção do funcionamento do consórcio e a transferência de recursos do SUS para o provimento dos serviços propriamente ditos. Adicionalmente, Teixeira et al. ${ }^{35}$ afirmam que o financiamento dessas estruturas se dá por três principais fontes: quotas dos municípios (definidas segundo critérios populacionais e/ou utilização dos serviços), recursos provenientes diretamente do SUS e recursos provenientes das Secretarias Estaduais de Saúde.

Galindo et al. ${ }^{18}$ destacam os dois instrumentos que formalizam a transferência de recursos para os consórcios: contrato de rateio e contrato de programa. Para os autores, a partir do contrato de programa é que o Consórcio passa a executar os serviços públicos demandados pela sociedade. O contrato de rateio, por usa vez, define
Tabela 1. Número de consórcios de saúde por estado.

\begin{tabular}{cc}
\hline Estado & Número de Consórcios \\
\hline MG & 68 \\
PR & 23 \\
SP & 23 \\
CE & 21 \\
SC & 16 \\
RS & 16 \\
MT & 15 \\
PB & 10 \\
RJ & 8 \\
ES & 7 \\
RN & 3 \\
PA & 2 \\
AM & 1 \\
PE & 1 \\
BA & 1 \\
Interestadual & 1 \\
Total & 216 \\
\hline
\end{tabular}

Fonte: Observatório dos Consórcios Públicos e Federalismos ${ }^{47}$.

as responsabilidades econômico-financeiras por parte de cada consorciado e a forma de repasse de recursos para a realização das despesas do consórcio público, como encargos, gastos de pessoal ou bens necessários ao seu funcionamento.

No caso dos consórcios do Estado de Minas Gerais, Rocha e Faria ${ }^{34}$ indicam que a relação de igualdade entre os municípios se apresentou como um princípio presente na estruturação dos consórcios. Por isso, eles, em geral, se organizam a partir da criação de um Conselho de Municípios (em geral composto pelos secretários municipais de saúde), de um Conselho Fiscal (responsável pelo controle da gestão orçamentária e financeira) e de uma Secretaria Executiva. Botti ${ }^{36}$ , no caso do Consórcio Intermunicipal de Saúde da microrregião de Teles Pires (MT), observa que, para o desenvolvimento das suas ações, a organização utiliza equipes técnicas e administrativas oriundas dos municípios integrantes ou contratadas mediante seleção pública, sob o regime Consolidação das Leis do Trabalho (CLT).

Neves e Ribeiro ${ }^{31}$ ressaltam que a sustentabilidade desse modelo se dá por conta dos resultados políticos positivos que geralmente são obtidos. Os autores estudaram de forma aprofundada o caso do consórcio de saúde de Penápolis e perceberam que uma das razões para seu sucesso de longo prazo foi a estabilidade política permitida pela sucessão de mandatos de prefeitos e vereadores, além do bom relacionamento entre as ca- 
sas executiva e legislativa. Rocha e Faria ${ }^{34}$ ainda reforçam que o funcionamento dos consórcios do Estado de Minas Gerais depende das características da política municipal e que, em casos mais bem-sucedidos, os executivos buscam pautar suas decisões por critérios técnico-instrumentais, visando preservar a experiência contra ingerências político-eleitorais.

Morais e Chaves ${ }^{32}$ apontam, especificamente para o caso do Consórcio Intermunicipal de Saúde do Cerrado Araguaia Tocantins, que um obstáculo para o consórcio é a necessidade de superação de seus limites e fronteiras para a implementação e monitoramento das políticas públicas de saúde, repercutindo na dinâmica política, econômica e assistencial. Keinert et al. ${ }^{48}$ ainda destacam que há outros desafios a serem superados como a conscientização dos gestores municipais, escassez de recursos humanos, preocupação quanto à situação jurídica e o entendimento do Tribunal de Contas diretamente relacionado ao consórcio.

Além disso, embora sejam claros os benefícios de um consórcio, há ainda a existência de membros que se comportam como free riders, ou seja, que fazem uso do serviço público provido pelo consórcio, mas sem participar de seu financiamento, enfraquecendo assim o propósito original da associação ${ }^{49}$. Percebe-se, ainda, que consórcios compostos por municípios mais homogêneos em termos de população, recursos financeiros e poder político, parecem ter maiores condições de dar certo, uma vez que, quando há assimetrias, os municípios mais fortes tendem a capitalizar para si os efeitos positivos da cooperação ${ }^{34}$.

\section{Casos de Consórcios Intermunicipais de Saúde}

Experiências de grande relevância no consorciamento são as dos estados de Minas Gerais e do Paraná, principalmente pelo comprometimento que os governos desses estados tiveram com a criação dos consórcios ${ }^{5,34}$. No caso de Minas Gerais, verificou-se que a ação do governo estadual não se limitou à difusão do conceito e ao fornecimento de informações para a criação dos consórcios, mas também à vinculação da liberação de recursos estaduais ao consorciamento dos municípios, criando assim fortes incentivos para a formação dessas estruturas no estado.

Galindo et al. ${ }^{18}$ destacam o caso pernambucano e afirmam que, mesmo os consórcios sendo uma estratégia de gestão intermunicipal, a adoção dos consórcios públicos como estratégia de regionalização do SUS resultou de uma escolha do governo estadual. Os autores perceberam que tem sido uma tendência recente a participação do estado como financiador dos consórcios, através do repasse de verbas ou pela cessão de recursos humanos, materiais, equipamentos e apoio técnico.

Os consórcios do Estado do Paraná comprovam que sua formação foi fundamental para a melhoria do acesso a serviços especializados e para a ampliação da cobertura de atendimento ${ }^{48}$. No caso dos municípios que fazem parte do Consórcios de Saúde da Associação dos Municípios do Médio Vale do Itajaí, a aquisição de medicamentos conjunta possibilitou a redução dos custos, garantindo maior oferta de medicamentos e diminuição do desabastecimento do componente da Assistência Farmacêutica Básica, verificada através da redução na média de dias por medicamento em falta e o número de itens em falta9

No caso do Consórcio Paraná Saúde, percebeu-se que, pela comparação de preços entre o Banco de Preços do Ministério da Saúde e aqueles praticados pelo consórcio, os preços pagos pelo Consórcio para aquisição dos medicamentos foram sistematicamente mais baixos, e a variação observada esteve entre $0,7 \%$ até $68,0 \%$. O consórcio ainda tem uma grande cobertura populacional (88,2 \% dos municípios do estado e 55,6\% da população paranaense), indicando assim seu potencial como instrumento de promoção da equidade de acesso aos bens e serviços de saúde ${ }^{10}$.

No estado de São Paulo, destaca-se o caso do Consórcio de Saúde do Alto Vale do Ribeira (Cisavar), onde o consórcio permitiu ampliação da oferta dos serviços especializados, através do melhor aproveitamento dos serviços já existentes (diminuição da ociosidade do hospital-sede), além de diminuir o comportamento free rider dos municípios vizinhos, que passaram a contribuir com recursos financeiros para o hospital ao qual já encaminhavam pacientes ${ }^{43}$. A autora ainda ressalta que houve o aumento do poder de barganha dos municípios envolvidos junto ao governo estadual, levando recursos à região que dificilmente seriam obtidos caso os solicitassem isoladamente.

A descentralização da atenção e da gestão dos recursos e das ações e serviços em saúde no Estado do Mato Grosso foi iniciada a partir de 1995, através do processo de articulação e cooperação técnica com os municípios, sendo o governo estadual o responsável por esta condução ${ }^{36}$. A autora aponta que um estudo realizado pela Secretaria Estadual de Saúde, entre os anos de 1995 e 
2000, quanto ao desempenho dos consórcios de saúde da região mostrou um aumento da resolução regional na assistência ambulatorial e hospitalar, verificado através da redução dos encaminhamentos de procedimentos dos municípios do estado para a capital Cuiabá e para outros estados da União.

No caso do Consórcio Intermunicipal de Saúde da Região do Teles Pires (MT), observase que os problemas não solucionados pelo consórcio foram relativos ao sistema de referência e contrarreferência e à existência de filas para a realização de procedimentos eletivos e para consultas em algumas especialidades. Por conta disso, verificou-se a importância de realização de estudos para mapeamento da real demanda, além de projeções futuras dessa demanda, para que haja um melhor planejamento dos consórcios quanto à sua oferta de serviços e definição quanto à sua capacidade de atendimento ${ }^{44}$.

Como contraexemplos, pode-se citar os estados das regiões Norte e Nordeste, que são as que têm menores recursos de serviços de especialidades, hospitais e assessoria técnica especializada, e mesmo assim não utilizam amplamente o modelo de consórcios de saúde ${ }^{43}$. A autora destaca dois fatores que podem inibir a cooperação municipal nesses casos. A primeira razão seria a extensão territorial dos municípios (muito grande, no caso da região Norte), distantes entre si e de difícil acesso, o que dificulta o trânsito de pessoas para acessar os serviços da região. O segundo fator se justifica por se tratar de regiões muito pobres e que, por isso, podem não contar com um patamar mínimo de serviços desejável, dado que não possuem os requisitos básicos em termos de equipamentos e recursos de saúde. $\mathrm{Ou}$ seja, o consorciamento não produziria ganhos adicionais para os municípios envolvidos em termos de serviços disponibilizados aos cidadãos. No entanto, esses municípios, ao se unirem no entorno de um consórcio, poderiam ampliar seu poder de barganha junto aos governos estadual e federal para a captação de recursos para saúde.

Teixeira ${ }^{38}$ acredita que os estados podem ocupar uma posição mais proativa no processo de formação e de manutenção dos CIS através de dois mecanismos complementares. Eles podem estimular a formação das parcerias ao facilitar para os consórcios a adoção de tecnologias inovadoras, que de outra forma seriam inacessíveis por seu elevado custo. Além disso, o estado pode também garantir que os membros de um consórcio não se tornem inadimplentes, mediante um contrato entre os municípios e o estado, que as- seguraria a transferência de recursos diretamente aos consórcios em caso de descumprimento das cláusulas relacionadas a repasses financeiros.

\section{Comentários finais}

As formas de relacionamento intergovernamental e, mais especificamente, dos consórcios intermunicipais, é um tema ainda pouco explorado pela ciência no que tange às políticas públicas ${ }^{43}$. Para o autor, mesmo sendo um assunto relevante, os estudos sobre os consórcios de saúde encontram grande dificuldade para aprofundar os seus achados em função da falta de dados históricos, em especial para os pequenos municípios, que são os que mais se consorciam, o que dificulta a comprovação dos bons resultados que esse tipo de arranjo pode proporcionar.

Adicionalmente, em tentativas de racionalização dos gastos com saúde, as ações centradas na redução dos gastos relacionados com os elos de fornecimento de insumos e serviços para os sistemas de saúde têm se mostrado mais eficazes do que ações de restrição de acesso aos demandantes desses serviços ${ }^{50}$. Além disso, os consórcios intermunicipais de saúde têm apresentado vantagens no que tange ao custo de aquisição e/ou contratação de serviços e à redução das faltas desse insumo e disponibilidade de serviços ${ }^{5,9,10,34,48}$. Desta forma, ações focadas na estruturação da relação entre os Sistemas de Saúde e os fornecedores de insumos e prestadores de serviços são justificadas dado seu potencial de melhoria na eficiência de serviços de saúde.

Ainda explorando o argumento da importância da racionalização dos gastos em saúde, um indicador intensamente utilizado pelas nações é a avaliação do percentual do Produto Interno Bruto (PIB) que se destina a ações em saúde. De acordo com o Global Health Observatory ${ }^{51}$, instituto ligado à Organização Mundial da Saúde para o monitoramento e comparação dos indicadores de saúde entre os países, o Brasil, em 2013, dedicou 9,7\% de seu PIB para a saúde e 6,9\% dos gastos totais do Governo.

Embora apresentem em seu sistema de saúde os princípios da integralidade e universalidade, é possível inferir que estes objetivos ainda estão longe de serem alcançados dado que os gastos do Governo representam apenas 48,2\% dos gastos totais em saúde no Brasil, demonstrando que uma grande proporção deste dispêndio é realizada diretamente, de forma privada, pelas famílias. Estes dados comprovam, quantitativamente, 
que há a necessidade de melhoria do Sistema de Saúde Brasileiro, para que ele atinja seu objetivo de integralidade e universalidade, e isso passa por melhorias nos mecanismos de compra de insumos e contratação de serviços.

Do ponto de vista acadêmico-científico, a revisão bibliográfica permitiu verificar que há um grande número de estudos que discutem o contexto histórico e jurídico da implantação dos consórcios no Brasil. Além disso, há estudos que se focam no entendimento sobre a formação ou o desempenho de um único consórcio intermunicipal (estudo de caso único) mas poucos têm o propósito de realizar uma comparação entre dois ou mais consórcios em uma mesma região. Há ainda estudos que tratam sobre avaliação de desempenho dos consórcios, mas as questões relacionadas com a governança dos consórcios são apontadas apenas como um dos fatores que podem afetar esse desempenho, sem que haja um detalhamento sobre essas estruturas e mecanismos.
Do ponto de vista da saúde pública, essa pesquisa permitiu observar que há ganhos evidentes com a adoção dos consórcios intermunicipais de saúde, em especial para os pequenos e médios municípios, e que os governos estaduais podem se apresentar como fortes indutores dessa estratégia. No entanto, um desafio para a efetiva implementação dos consórcios está na governança dessas organizações, dada a variedade de atores envolvidos nesse processo decisório.

No entanto, é possível verificar que, no universo pesquisado, há uma lacuna de trabalhos e publicações que tentam compreender, de forma detalhada e aprofundada, as estruturas e mecanismos de governança dos consórcios. Em se tratando de estruturas organizacionais onde a tomada de decisão se dá envolvendo diversos prefeitos e secretários de saúde, com possíveis orientações políticas distintas e prioridades que não são necessariamente convergentes, estudos sobre a governança dessas organizações mostram-se como um desafio para o avanço dos estudos acadêmicos sobre o tema.

\section{Colaboradores}

RGC Flexa realizou a concepção e o desenho do estudo, bem como as análises. RG Barbastefano contribuiu com a seleção dos artigos, bem como na redação e a revisão do texto final. 


\section{Referências}

1. Santos I. O Mix Público-Privado no Sistema de Saúde Brasileiro: elementos para a regulação da cobertura duplicada [tese]. Rio de Janeiro: Escola Nacional de Saúde Pública; 2009.

2. Biasoto Júnior G, Silva P, Dain S. Regulação do setor saúde nas Américas: as relações entre o público e o privado numa abordagem sistêmica. Brasília: OPAS; 2006.

3. Conselho Nacional de Secretários de Saúde (CONASS). Para entender a gestão do SUS: a gestão do SUS. Brasília: CONASS; 2015.

4. Buss P. A IX Conferência Nacional de Saúde. Cad Saude Publica 1991; 7(3):297-300.

5. Souto Júnior JV. O papel da CIB/MG no processo de regionalização do SUS em Minas Gerais [dissertação]. Rio de Janeiro: Escola Nacional de Saúde Pública; 2010.

6. Kehrig RT, Souza ES, Scatena JHG. Institucionalidade e governança da regionalização da saúde: o caso da região Sul Mato-Grossense à luz das atas do colegiado de gestão. Saúde Debate 2015; 39(107):948-961.

7. Conselho Nacional de Secretários de Saúde (CONASS). Para entender a gestão do SUS. Brasília: CONASS; 2011.

8. Baldissera DS. Consórcios Públicos Intermunicipais no Brasil: Panorama após os 10 anos da Lei 11.107/2005 [dissertação]. Rio de Janeiro: FGV; 2015.

9. Amaral SMS, Blatt CR. Consórcio intermunicipal para a aquisição de medicamentos: impacto no desabastecimento e no custo. Rev Saude Publica 2011; 45(4):1-3.

10. Ferraes AMB, Cordoni Júnior L. Consórcio de medicamentos no Paraná: análise de cobertura e custos. Revista de Administração Pública 2007; 41(3):475-486.

11. Gil A. Como elaborar projetos de pesquisa. São Paulo: Atlas; 2008.

12. Rother ET. Revisão sistemática X revisão narrativa. Acta Paulista de Enfermagem 2007; 20(2):vii-viii.

13. Observatório dos Consórcios Públicos e do Federalismo. Federalismo. [Online]. 2017. [acessado $2017 \mathrm{Mar}$ 15]. Disponível em: http://www.ocpf.org.br/federalis$\mathrm{mo} /$

14. Vinhas LP, Tupinambá JCD. Cooperação intergovernamental em busca do desenvolvimento regional. In: Anais do VI Congresso de Gestão Pública - CONSAD; 2011; Brasília. p .3-30

15. Trevas V. Consórcios Públicos e o Federalismo Brasileiro. Projetos para o Brasil: Consórcios Públicos e as Agendas do Estado brasileiro. São Paulo: Fundação Perseu Abramo; 2013.

16. Batista S, Vecchia R, Pereira MP, Augusti MT, Hernandes JL, Bachiega CC, [acrescentar os demais autores]. O papel dos dirigentes municipais e regionais na criação e gestão dos consórcios públicos. Brasília: Caixa Econômica Federal; 2011.

17. Brasil. Lei no 11.107, de 6 de abril de 2005. Dispõe sobre normas gerais de contratação de consórcios públicos e dá outras providências. Diário Oficial da União 2005; 7 abr.

18. Galindo JM, Cordeiro JC, Villani RAG, Barbosa Filho RA, Rodrigues CS. Gestão interfederativa do SUS: a experiência gerencial do Consórcio Intermunicipal do Sertão do Araripe de Pernambuco. Rev. Adm. Pública. 2014; 48(6):1545-1566.
19. Linhares PS, Mendes CS, Lassance A, organizadores. Federalismo à brasileira: questões para discussão. Brasília: IPEA; 2012.

20. Ramirez AG, Tracci MC, Stukenborg GJ. Physician-Owned Surgical Hospitals Outperform Other Hospitals in Medicare Value-Based Purchasing Program. J Am Coll Surg 2016; 223(4):559-567.

21. Sorenson C, Kanavos P. Medical technology procurement in Europe: A cross-country comparison of current practice and policy. Health Policy 2011;100(1):4350 .

22. Burns L, Lee J. Hospital purchasing alliances: Utilization, services, and performance. Health Care Manage Rev 2008; 33(3):203-215.

23. Hansson J, Ovretveit J, Brommels M. Case study of how successful coordination was achieved between a mental health and social care service in Sweden. Int $J$ Health Plann Manage 2012; 27(2):132-145.

24. Datta D, Figueira JR, Gourtani AM, Morton A. Optimal administrative geographies: An algorithmic approach. Socio-Economic Planning Sciences 2013; 47(3):247-257.

25. Chakraborty S, Bhattachary S, Dobrzykowski DD. Impact of Supply Chain Collaboration on Value Co-creation and Firm Performance: A Healthcare Service Sector Perspective. Procedia Economics and Finance 2014; 11(2014):676-694.

26. Mathew J, John J, Kumar S. New Trends in Healthcare Supply chain. In: Annals of POMS Conference Proceedings; Denver; 2013. p. 1-10.

27. Reid PP, Compton W, Grossman J, Fanjiang G. Building a Better Delivery System: A New Engineering/ Health Care Partnership. Washington: The National Academies Press; 2005.

28. Hinrichs S, Jahagirdar D, Miani C, Guerin B, Nolte E. Learning for the NHS on procurement and supply chain management: a rapid evidence assessment. Health Services and Delivery Research 2014; 2(55):1158.

29. Pepino A, Rovani M, Torri A, Sansone M. Supply Chain for Regional Centralized Purchasing: An Application Model. Intelligent Information Management 2012; 4(5A):269-276.

30. Lagarde M, Haines A, Palmer N. Conditional Cash Transfers for Improving Uptake of Health Interventions in Low- and Middle-Income Countries. JAMA 2007; 298(16):1900-1910.

31. Neves LA, Ribeiro JM. Consórcios de saúde: estudo de caso exitoso. Cad Saude Publica 2006; 22(10):22072217.

32. Morais VS, Chaves APL. Percepção dos gestores municipais de saúde relacionada à saúde ambiental: consórcio intermunicipal de saúde Cerrado Tocantins Araguaia. Saúde Soc. 2016; 25(2):349-360.

33. Chaebo G, Guerra M, Pinto D, Alfinito S. Constituição de Consórcios Intermunicipais de Saúde: Uma Aplicação da Técnica de Agrupamento por Clusters. Latin American Journal of Business Management. 2015; 6(2):149-169.

34. Rocha CV, Faria CAP. Cooperação intermunicipal, reterritorialização da gestão pública e provisão de bens e serviços sociais no Brasil contemporâneo: experiência dos consórcios de saúde em Minas Gerais. Cadernos Metrópole 2004; 11:73-105. 
35. Teixeira L, Mac Dowell MC, Bugarin M. Consórcios Intermunicipais de Saúde: Uma Análise à Luz da Teoria dos Jogos. Rev. Bras. Econ. 2003; 57(1):253-281.

36. Botti CS. Avaliação do processo de implementação do Consórcio Intermunicipal de Saúde da região do Teles Pires [dissertação]. Rio de Janeiro: Escola Nacional de Saúde Pública; 2010.

37. Rodrigues A. Cooperação intermunicipal no âmbito do SUS [dissertação]. São Paulo: USP; 2003.

38. Teixeira L. Ensaios sobre consórcios intermunicipais de saúde: financiamento, comportamento estratégico, incentivos e economia política. Brasília: Câmara dos Deputados/CODEP; 2007.

39. Martinelli N. A regionalização da saúde no Estado do Mato Grosso: o processo de implementação e a relação público-privada na região de saúde do Médio Norte mato-grossense [tese]. São Paulo: USP; 2014.

40. Strelec TC. Desafios da Adaptação à Lei de Consórcios Públicos: experiências concretas no Estado de São Paulo. In: Anais do VI Congresso de Gestão Pública CONSAD; 2011; Brasília. p. 2-20.

41. Cruz MCMT, Araújo FF. Consórcios intermunicipais paulistas rumo aos consórcios públicos - reflexões. In: Anais do VI Congresso de Gestão Pública - CONSAD; 2011; Brasília. p. 2-20

42. Muller EV, Grecco M. Avaliação da satisfação dos usuários com os serviços do consórcio intermunicipal de saúde do noroeste do Paraná. Cien Saude Colet 2010; 15(3):925-930.

43. Oliveira VE. Municípios cooperando com municípios: relações federativas e consórcios intermunicipais de saúde no Estado de São Paulo. São Paulo em Perspectiva 2008; 2(1):107-122.

44. Botti CS, Artmann E, Spinelli MAS, Scatena JHG. Regionalização dos Serviços de Saúde em Mato Grosso: um estudo de caso da implantação do Consórcio Intermunicipal de Saúde da Região do Teles Pires, no período de 2000 a 2008. Epidemiol. Serv. Saúde 2013; 22(3):491-500.

45. Brasil. Constituição da República Federativa do Brasil Brasília: Imprensa Nacional; 1988.
46. Batista S. O papel dos prefeitos e das prefeitas na criação e na gestão dos consórcios públicos. Brasília: Caixa Econômica Federal; 2011.

47. Observatório dos Consórcios Públicos e do Federalismo. Banco de Dados. [Online].; 2016. [acessado 2016 Out 15]. Disponível em: http://www.ocpf.org.br/consorcios-publicos/banco-de-dados/.

48. Keinert TM, Meneguzzo M, Rosa TC. Inovação e cooperação intergovernamental: microrregionalização, consórcios, parcerias e terceirização no setor saúde. São Paulo: Annablume; 2006.

49. Chaebo G, Guerra M, Pinto DM, Alfinito S. Constituição de Consórcios Intermunicipais de Saúde: Uma Aplicação da Técnica de Agrupamento por Clusters. In: Anais do EnAPG; 2012; Salvador. p. 1-16

50. Blank R, Burau V. Comparative Health Policy. London: Palgrave Macmillan; 2007.

51. Global Health Observatory. Databases. [Online]; 2016 [acessado 2016 Out 15]. Disponível em: http://www. who.int/gho/en/.

Artigo apresentado em 22/07/2017

Aprovado em 22/05/2018

Versão final apresentada em 24/05/2018 